Research Article

\title{
Willingness to Adopt Telemedicine in Major Iraqi Hospitals: A Pilot Study
}

\author{
Mohd Khanapi Abd Ghani and Mustafa Musa Jaber \\ Biomedical Computing and Engineering Technologies (BIOCORE) Applied Research Group, \\ Faculty of Information and Communication Technology, Universiti Teknikal Malaysia Melaka, Durian Tunggal, \\ 75450 Ayer Keroh, Malacca, Malaysia \\ Correspondence should be addressed to Mustafa Musa Jaber; mustafamusajaber@yahoo.com
}

Received 21 May 2015; Revised 20 August 2015; Accepted 13 September 2015

Academic Editor: Malcolm Clarke

Copyright ( 2015 M. K. Abd Ghani and M. M. Jaber. This is an open access article distributed under the Creative Commons Attribution License, which permits unrestricted use, distribution, and reproduction in any medium, provided the original work is properly cited.

\begin{abstract}
The Iraqi healthcare services are struggling to regain their lost momentum. Many physicians and nurses left Iraq because of the current situation in the country. Despite plans of calling back the skilled health workforce, they are still worried by the disadvantages of their return. Hence, technology plays a central role in taking advantage of their profession through the use of telemedicine. Studying the factors that affect the implementation of telemedicine is necessary. Telemedicine covers network services, policy makers, and patient understanding. A framework that includes the influencing factors in adopting telemedicine in Iraq was developed in this study. A questionnaire was distributed among physicians in Baghdad Medical City to examine the hypothesis on each factor. The Statistical Package for the Social Sciences was utilized to verify the reliability of the questionnaire and Cronbach's alpha test shows that the factors have values more than 0.7 , which are standard.
\end{abstract}

\section{Introduction}

Developing countries are using telemedicine to link health centers, tertiary centers, and referral hospitals. Several ehealth applications, such as telemedicine, can be found with different degrees of success in majority of developing countries [1,2]. According to [3], the following two factors explain the wide use of telemedicine: the lack of alternatives such as building hospitals in each village which is costly and (2) the apparent geographical superiority of telemedicine over traditional medicine. Despite high expectations from the technology, configuring its use into a certain routine is impossible [4].

The framework of adopting telemedicine in Iraq was categorized into four characteristics, namely, technologi$\mathrm{cal}$, organizational, environmental, and individual. Fourteen influencing factors were explicated, and each was included in its appropriate characteristic. This framework can enhance the quality of the healthcare sector in Iraq. Thus, the influencing factors are enablers in the decision-making and eparticipation of hospitals [5]. This study illustrates a suitable design of the questionnaire on willingness to adopt telemedicine of telemedicine in Iraq to evaluate the framework. A pilot study was conducted to improve the reliability of the questionnaire.

Several benefits can be expected from the use of telemedicine. These benefits can affect not only the health providers and the patients, but also the entire healthcare organization. For example, a study on physicians and health managers in the Quebec health region in Canada by [6] found that the benefits and usefulness of the telemedicine system were seen on three levels: (1) clinical/patient, (2) professional/educational, and (3) organizational.

\section{Limitations of the Research}

This study aims to examine the feasibility and acceptance level telemedicine framework in Iraq. However, a number of limitations should be considered. First, studies regarding telemedicine in Iraq are limited, with few studies conducted by the Ministry of Health of Iraq. This situation caused 
some difficulties in designing the questionnaire. Second, the physicians' level of understanding of telemedicine is limited because telemedicine is a new technology in Iraq $[5,7]$. Third, only a handful of physicians practice electronic consultation (e-consultation). Fourth, physicians have limited grasp and knowledge of data warehouse because it is viewed and considered as a new tool in telemedicine. Fifth, the number of respondents was 35 only. Finally, the unstable political climate and strife in Iraq added challenges to the scope and limitations of this study.

\section{The Framework}

The technology-organization-environment (TOE) framework, which was developed by [8], is chosen and applied as the framework of this study. [1, 2], These studies have demonstrated consistent support for TOE's ability to provide a comprehensive perspective on innovation adoption, while facilitating the flexibility to identify and categorize unique factors that may emerge in particular situations [9]. Moreover, the main reason for selecting this framework is that this approach has the potential to address issues of this study. On the subject of appropriate framework selection, [10] advocates a framework that has been adapted and adopted and requires further development and fine-tuning in its application for contextual matching.

This study adapted the TOE framework to improve its suitability in the field of telemedicine. This adaption considers technological, organizational, environmental, and individual characteristics. In addition, social exchange theory, diffusion of innovation theory, TOE adoption framework, and previous studies are employed to investigate the factors that influence telemedicine in Iraq. These theories have been applied in electronic information sharing studies [11, 12]. Social exchange theory refers to shared information in the public sector [13]. This theory is based on power and trust. Thus, factors such as top management support of upper-level leadership are employed. The focus of this theory is to help organizations and individuals in deciding whether to adopt or reject a new innovation and to estimate the duration of acceptance and use of a new technology. In reality, the theory has been used to clarify and evaluate a wide range of IT adoption, such as that of the Internet [14], database machine [15], software engineering techniques [16], and IT in general [17].

The remaining factors have been adopted from researches done in Middle Eastern countries such as Kuwait and Jordan. Each characteristic has numerous influencing factors. The influencing factors of the current research are privacy, culture, attitude to telemedicine, benefit, connectivity, IT capability, compatibility, data warehouse concept, technical support, top management support, cost, approach, and upperlevel leadership. This study also introduces 13 hypotheses. One hypothesis is formulated for every factor. Figure 1 shows the framework of this study.

The factors that can promote engagement in the willingness to accept telemedicine have been identified. The pilot study is performed to evaluate the questionnaire items and to determine the appropriate questions for the survey.

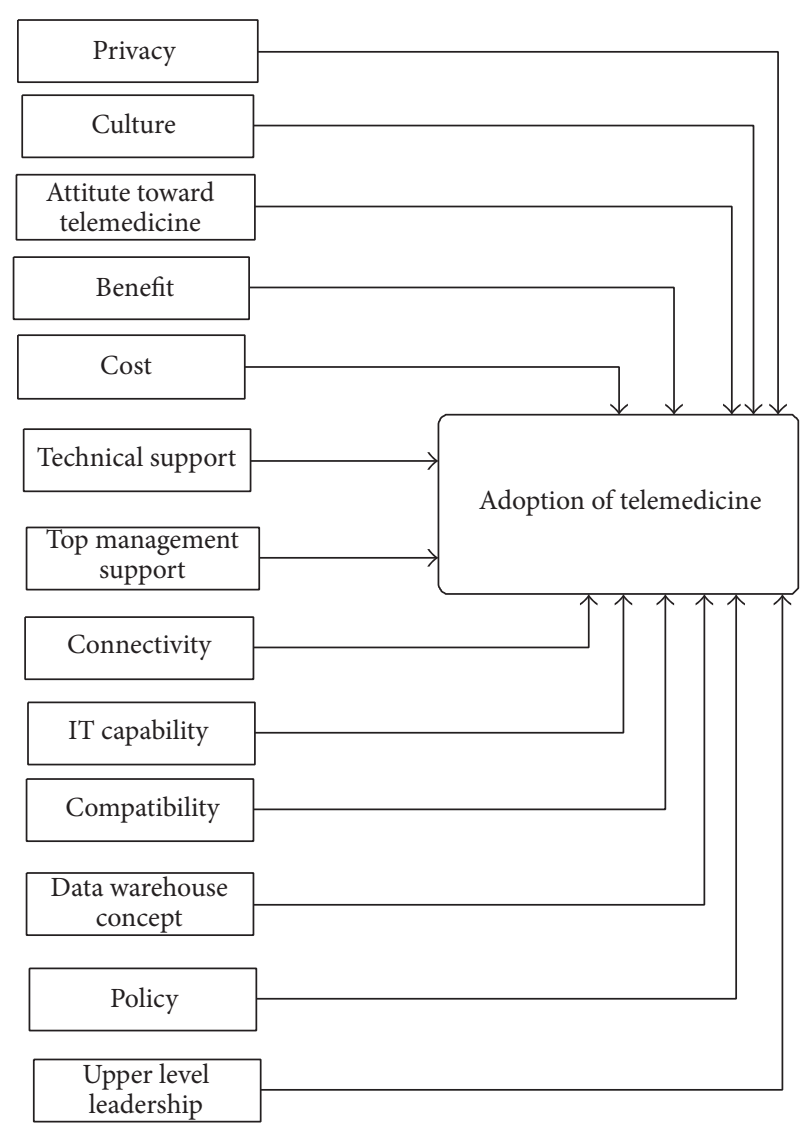

FIGURE 1: Framework of willingness to adopt telemedicine in Iraq.

The questionnaires were distributed among physicians. The succeeding section explains the questionnaire design.

\section{Research Methods}

The survey method is employed in this study because surveys are prevalently used to begin reports and it is a suitable method for examining factors and hypotheses. The questionnaire methodology is used for data collection. Paper [18] shows that the questionnaire design relies on three criteria, namely, the manner by which the questions are written, planning for the classification of variables, and the appearance of the questionnaire. This study uses a six-part questionnaire. Part 1 includes the questions related to demographic factors. Part 2 relates to the state of telemedicine. Part 3 relates to the characteristics of telemedicine. Part 4 relates to organizational characteristics. Part 5 includes questions related to technological characteristics. Part 6 relates to environmental characteristics. Please refer to the Appendix.

The questionnaire is designed based on the content of each factor. Suggestions and advice from colleagues and supervisors are considered in improving the design of the questionnaire, as well as in developing the questionnaire. The questionnaire is written in English and then translated into Arabic, which is the official language in Iraq. Table 1 shows the operationalization of the factors and items. 
TABLE 1: Operationalization of the factors and items.

\begin{tabular}{lcc}
\hline Factor & Items & References \\
\hline Privacy & 3 items & {$[17,19]$} \\
Culture & 3 items & {$[19-21]$} \\
Attitude toward telemedicine & 3 items & {$[22,23]$} \\
Benefit & 5 items & {$[20,21,24]$} \\
Connectivity & 3 items & {$[25,26]$} \\
IT capability & 3 items & {$[20,21,24,27]$} \\
Compatibility & 3 items & {$[5,13,28]$} \\
Data warehouse & 3 items & {$[29-31]$} \\
Technical support & 3 items & {$[21,32]$} \\
Top management support & 3 items & {$[5,13,28,33]$} \\
Cost & 4 items & {$[20,24,34]$} \\
Policy & 3 items & {$[5,13,20,21,28]$} \\
Upper-level leadership & 3 items & {$[5,13,35]$} \\
\hline
\end{tabular}

TABle 2: Demographic characteristics.

\begin{tabular}{lcc}
\hline & \multicolumn{2}{c}{$\%$ of participants } \\
& Pilot study 1 & Pilot study 1 \\
\hline Variable & 24 & \\
Male & 6 & $80.0 \%$ \\
Female & 3 & $20.0 \%$ \\
Less than 30 & 21 & $10.0 \%$ \\
From 30 to 40 & 5 & $70.0 \%$ \\
From 41 to 50 & 1 & $16.7 \%$ \\
More than 50 & 21 & $3.3 \%$ \\
Bachelor & 8 & $70.0 \%$ \\
Master's & 1 & $26.7 \%$ \\
Ph.D. & & $3.3 \%$ \\
Experience in years & 6 & \\
From 1 to 5 & 14 & $20.0 \%$ \\
From 6 to 10 & 8 & $46.7 \%$ \\
From 11 to 15 & 2 & $26.7 \%$ \\
$\quad$ More than 15 & & $6.7 \%$ \\
Type of position & 12 & \\
Administrator & 18 & $60.0 \%$ \\
Nonadministrator & &
\end{tabular}

\section{Pilot Study}

Questionnaires were distributed among physicians who are working in Baghdad Medical City. Among the 35 questionnaires collected, only 5 were not correctly answered. Table 2 illustrates the demographic characteristics of the pilot study.

5.1. Usage of Devices. Queries on the method of how physicians conduct electronic consultation in inputting patient information were conducted in this study. Five methods were mentioned in the questionnaire, but the participants were provided an option to add another method that they have used. However, no other method of information sharing was added by the participants. Thus, each study reveals different

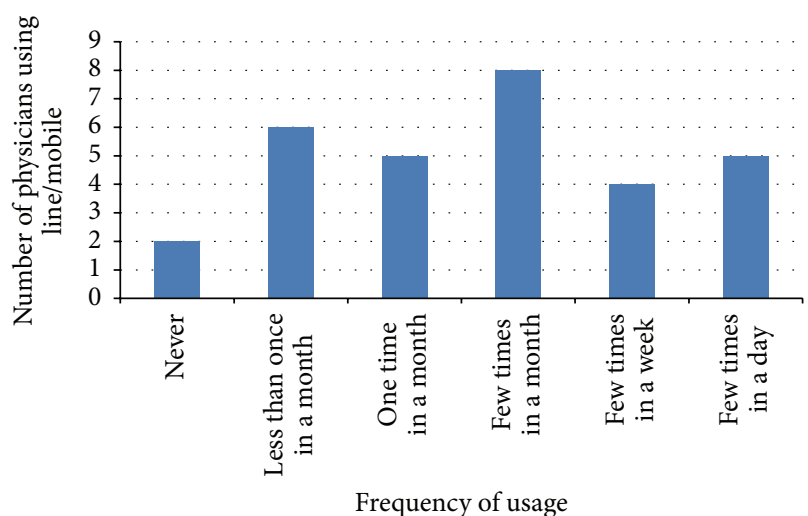

Figure 2: Line/mobile usage.

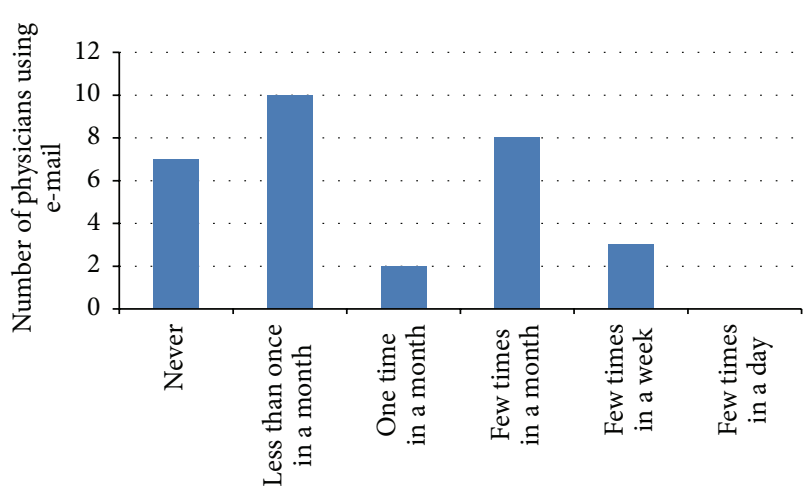

Frequency of usage

Figure 3: E-mail usage.

percentages related to the five methods used. Figures 2, 3, 4, and 5 illustrate the percentages of the most used method of electronic consultation. The data collected reveal that e-mail is the most used method in electronic consultation.

The database was not utilized by the participants to access the ministry's information. The results from the two pilot studies reveal that the most used means of electronic consultation between physicians and patients are phone, email, and website. The findings also show that the use of webcam is limited. The use of database was mentioned only once in both studies.

5.2. Percentage and Initiation Time of Sharing Information. The electronic information sharing practice in the Iraqi healthcare sector was measured in terms of the percentage of electronic consultation used and the time information consultation was initiated. Thus, based on the findings, the participants of the pilot studies illustrated the percentage of electronic consultation used. Most of the participants revealed that the percentage of consultation is $63.3 \%$ from $1 \%$ to $20 \%$ of their work. Most of the participants mentioned that electronic consultation was initiated between 1 year and 3 years ago. Table 3 shows the consultation percentage per year was initiated. 


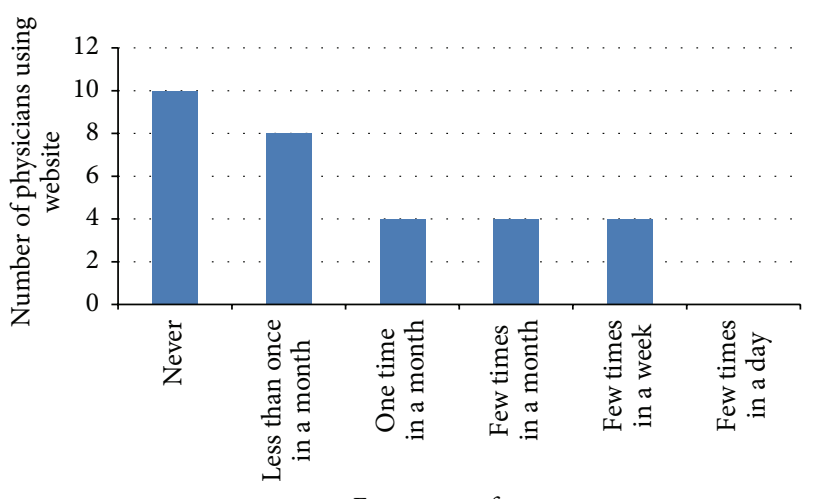

Frequency of usage

Figure 4: Website usage.

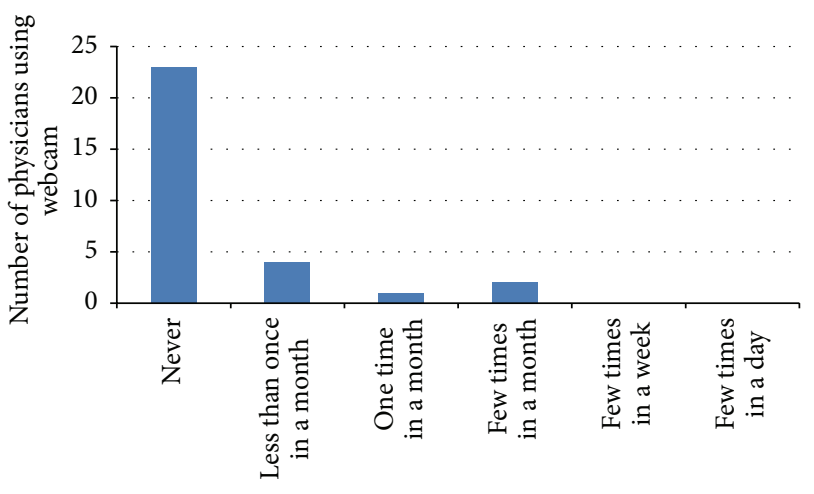

Frequency of usage

Figure 5: Webcam usage.

TABLE 3: Percentage and start/initiation time of consultation.

\begin{tabular}{lcc}
\hline Variables & Number of participants $\%$ of participants \\
\hline Percentage of consultation & 5 & $16.7 \%$ \\
Zero & 19 & $63.3 \%$ \\
From $1 \%$ to $20 \%$ & 5 & $16.7 \%$ \\
From $21 \%$ to $40 \%$ & 1 & $3.3 \%$ \\
From $41 \%$ to $60 \%$ & 0 & $0.0 \%$ \\
From $61 \%$ to $80 \%$ & 0 & $0.0 \%$ \\
From $81 \%$ to $100 \%$ & & \\
Years of consultation & 1 & $3.3 \%$ \\
Zero & 9 & $30.0 \%$ \\
Less than a year & 18 & $60.0 \%$ \\
From 1 to 3 years & 2 & $6.7 \%$ \\
From 4 to 6 years & 0 & $0.0 \%$ \\
From 7 to 9 years & 0 & $0.0 \%$ \\
10 years or more & & \\
\hline
\end{tabular}

\section{Results of Data Analysis}

The most commonly used test in reliability measurement of any pilot study questionnaire is Cronbach's alpha [18, 36]. According to [37], Cronbach's alpha test possesses values
TABLE 4: Cronbach's alpha and number of items.

\begin{tabular}{lcc}
\hline Factor & Cronbach's alpha & Number of items \\
\hline Privacy & .756 & 3 \\
Culture & .926 & 3 \\
Attitude toward telemedicine & .755 & 3 \\
Benefit & .729 & 5 \\
Connectivity & .794 & 3 \\
IT capability & .706 & 3 \\
Compatibility & .763 & 3 \\
Data warehouse & .749 & 3 \\
Technical support & .787 & 3 \\
Top management support & .706 & 3 \\
Cost & .728 & 4 \\
Policy & .815 & 3 \\
Upper-level leadership & .818 & 3 \\
\hline
\end{tabular}

within the range of 0 to 1 ; a higher level of range indicates greater value of reliability. Values of 0.9 and above are excellent, 0.8 and above are good, 0.7 and above are acceptable, 0.6 and above are questionable, and less than 0.6 are poor $[37,38]$.

The data collected from the two pilot studies have been analyzed using Statistical Package for the Social Sciences 20 to identify the values of each factor in Cronbach's alpha. Based on the pilot study, no change should be made in the items of the pilot study. These items were rewritten to create a clear and easy-to-understand questionnaire. The items of the factors were also reduced. Thus, all factors have values of more than 0.7 , which are acceptable. Table 4 shows Cronbach's alpha and number of items for each factor.

\section{Conclusion and Future Work}

This study was proposed to examine the influencing factors in the willingness to adopt and practice telemedicine in Iraq. This study was prompted by a real need to examine the requirements, challenges, and gaps in adopting this new technology. A set of items of questionnaires was designed based on previous studies on the adoption of telemedicine in the Middle Eastern countries, such as Kuwait, Saudi Arabia, Jordan, and Syria. The pilot studies were conducted to examine each influencing factor in the hypotheses and to test the reliability of the questionnaire. The pilot study was required to verify the items. Cronbach's alpha test in the pilot study reveals that the factors have values more than 0.7 , which are acceptable. The pilot study was conducted using questionnaires that were disseminated to physicians in Iraq. A research paper will follow this study to illustrate the results of the data analysis of the survey. Future research will then test the hypotheses and validate the framework. The results of the tests are expected to contribute to furthering the understanding and grasp of electronic information sharing in the healthcare sector. In addition, research of willingness to adopt telemedicine needs to be examined in rural area since it has different factors to measure. 
TABle 5: Part 3: individual characteristic.

\begin{tabular}{l}
\hline \\
$\begin{array}{l}\text { Pril: being concerned about patient privacy is important. } \\
\text { Pri2: we have to keep the privacy of patients. }\end{array}$ \\
Pri3: telemedicine is not private and confidential. \\
Cul1: applying telemedicine will be negatively affected by culture and \\
religion issues. \\
Cul2: social issues (culture and religion) have a potential effect on \\
applying telemedicine. \\
Cul3: our culture and social norms refuse the use of telemedicine. \\
ATT1: I trust technology to work. \\
ATT2: I am happy using ICT/the Internet for patient care. \\
ATT3: general comfort exists in using ICT/Internet to store, retrieve, \\
and communicate patient information with other health institutions. \\
Ben1: telemedicine enhances collaboration in the public sector. \\
Ben2: telemedicine improves the healthcare service quality in Iraq. \\
Ben3: telemedicine reduces cost in overall healthcare expenses. \\
Ben4: telemedicine improves efficiency and resource utilization. \\
Ben5: telemedicine improves access to healthcare services and care \\
delivery, especially for people in rural and remote communities.
\end{tabular}

TABLE 6: Part 4: technological characteristic.

\begin{tabular}{l}
\hline \\
Con1: the connectivity is important for healthcare delivery. \\
Con2: physicians have to be connected to the Internet. \\
Con3: physicians have to be connected to patients. \\
ITc1: our healthcare requires information system applications. \\
ITc2: our healthcare requires good ICT infrastructure. \\
ITc3: telemedicine requires basic IT skills. \\
Compat1: physician ICT is different from patient ICT. \\
Compat2: telemedicine does not contrast with healthcare needs. \\
Compat3: different telemedicine applications will not affect the \\
efficiency. \\
DWc1: we should keep patient health record perpetually. \\
DWc2: there are needs of patient health record to have statistic. \\
DWc3: we should store our patient's record in a common database to \\
make them accessible.
\end{tabular}

TABle 7: Part 5: organization characteristic.

\begin{tabular}{|c|c|c|c|c|c|}
\hline & Strongly disagree & Disagree & Neutral & Agree & Strongly agree \\
\hline \multicolumn{6}{|l|}{ TS1: telemedicine requires good technical support. } \\
\hline \multicolumn{6}{|l|}{$\begin{array}{l}\text { TS2: a specific focus on long-term sustainability is a common trait } \\
\text { among successful programs. }\end{array}$} \\
\hline \multicolumn{6}{|l|}{$\begin{array}{l}\text { TS3: technical support provides measurement and feedback to } \\
\text { physicians. }\end{array}$} \\
\hline \multicolumn{6}{|l|}{ TMS1: our top managers are interested in applying telemedicine. } \\
\hline \multicolumn{6}{|l|}{ TMS2: our top managers have properly identified telemedicine needs. } \\
\hline \multicolumn{6}{|l|}{ TMS3: our top managers have prioritized telemedicine needs. } \\
\hline \multicolumn{6}{|l|}{$\begin{array}{l}\text { Cost 1: high cost of equipment might be the cause of not adopting } \\
\text { telemedicine. }\end{array}$} \\
\hline \multicolumn{6}{|l|}{ Cost 2: telemedicine is less costly than traditional healthcare delivery. } \\
\hline \multicolumn{6}{|l|}{ Cost 3: staff training is costly. } \\
\hline Cost 4: software and hardware maintenance is costly. & & & & & \\
\hline
\end{tabular}


TABLE 8: Part 6: environmental characteristic.

\begin{tabular}{l}
\hline \\
\begin{tabular}{l}
\hline Policy 1: the government should amend policies to support the \\
telemedicine project.
\end{tabular} \\
Policy 2: our hospitals require legislations and policies to apply the \\
telemedicine project. \\
Policy 3: legislations and policies build good relationship and trust \\
among our staff. \\
Upper 1: the Ministry of Health recommends the application of \\
telemedicine. \\
Upper 2: the Ministry of Health requests the application of \\
telemedicine. \\
Upper 3: the Ministry of Health provides the requirements to \\
implement telemedicine.
\end{tabular}

\section{Appendix}

\section{Factors and Items}

See Tables 5, 6, 7, and 8 and Figures 2 and 3.

\section{Conflict of Interests}

Mohd Khanapi Abd Ghani and Mustafa Musa Jaber have no conflict of interests regarding the publication of this paper.

\section{References}

[1] J. A. Blaya, H. S. F. Fraser, and B. Holt, "E-health technologies show promise in developing countries," Health Affairs, vol. 29, no. 2, pp. 244-251, 2010.

[2] M. M. Jaber, M. K. A. Ghani, and N. S. Herman, "A review of adoption of telemedicine in Middle East countries: toward building Iraqi telemedicine framework," Science International, vol. 26, no. 5, pp. 1795-1800, 2014.

[3] K. Schlein, A. Y. De La Cruz, T. Gopalakrishnan, and D. Montagu, "Private sector delivery of health services in developing countries: a mixed-methods study on quality assurance in social franchises," BMC Health Services Research, vol. 13, article 4, 2013.

[4] S.-H. Hsieh, S.-L. Hsieh, P.-H. Cheng, and F. Lai, "E-health and healthcare enterprise information system leveraging serviceoriented architecture," Telemedicine and e-Health, vol. 18, no. 3, pp. 205-212, 2012.

[5] M. K. A. Ghani, M. M. Jaber, and N. Suryana, "Telemedicine supported by data warehouse architecture," ARPN Journal of Engineering and Applied Sciences, vol. 10, no. 2, pp. 415-417, 2015.

[6] M.-P. Gagnon, G. Godin, C. Gagné et al., "An adaptation of the theory of interpersonal behaviour to the study of telemedicine adoption by physicians," International Journal of Medical Informatics, vol. 71, no. 2-3, pp. 103-115, 2003.

[7] M. A. Mohammed, M. H. Kadhim, A. Fuad, and M. M. Jaber, "Follow up system for directorate of scholarship and cultural relations in Iraq," in Proceedings of the International Conference on Computer, Communications, and Control Technology (I4CT '14), pp. 182-187, Langkawi, Malaysia, September 2014.

[8] L. G. Tornatzky, M. Fleischer, and A. K. Chakrabarti, Processes of Technological Innovation, Lexington Books, 1990.

[9] K. Zhu, K. Kraemer, and S. Xu, "Electronic business adoption by European firms: a cross-country assessment of the facilitators and inhibitors," European Journal of Information Systems, vol. 12, no. 4, pp. 251-268, 2003.

[10] S. Kurnia and R. B. Johnston, “The need for a processual view of inter-organizational systems adoption," The Journal of Strategic Information Systems, vol. 9, no. 4, pp. 295-319, 2000.

[11] A. Y. Akbulut, An investigation of the factors that influence electronic information sharing between state and local agencies [Ph.D. thesis], Louisiana State University, Baton Rouge, La, USA, 2003.

[12] A. Ziaee Bigdeli, M. M. Kamal, and S. de Cesare, "Electronic information sharing in local government: the decision-making process," in Proceedings of the 20th European Conference on Information Systems (ECIS '12), Barcelona, Spain, June 2012.

[13] R. M. Emerson, "Social exchange theory," Annual Review of Sociology, vol. 2, no. 1, pp. 335-362, 1976.

[14] N. Prammanee, "A critical analysis of the adoption and utilization of the internet in Thailand for education purposes," First Monday, vol. 8, no. 1, 2003.

[15] J. A. Hoffer and M. B. Alexander, "The diffusion of database machines," ACM SIGMIS Database, vol. 23, no. 2, pp. 13-19, 1992.

[16] J. Bayer and N. Melone, "A critique of diffusion theory as a managerial framework for understanding adoption of software engineering innovations," in Proceedings of the 21st Annual Hawaii International Conference on System Sciences, Volume II. Software Track, pp. 311-316, Kailua-Kona, Hawaii, USA, January1988.

[17] G. C. Moore and I. Benbasat, "Development of an instrument to measure the perceptions of adopting an information technology innovation," Information Systems Research, vol. 2, no. 3, pp. 192222,1991

[18] U. Sekaran and R. Bougie, Research Methods for Business: A Skill Building Approach, Wiley, London, UK, 2010.

[19] D. Ludwick and J. Doucette, "Primary care physicians' experience with electronic medical records: barriers to implementation in a fee-for-service environment," International Journal of Telemedicine and Applications, vol. 2009, Article ID 853524, 2009.

[20] A. Buabbas, Investigation of the adoption of telemedicine technology in the Kuwaiti health system: strategy and policy of implementation for overseas referral patients [Ph.D. thesis], School of Information Systems, Computing and Mathematics, Brunel University, Uxbridge, UK, 2013. 
[21] M. Alajlani and M. Clarke, "Effect of culture on acceptance of telemedicine in middle eastern countries: case study of Jordan and Syria," Telemedicine and e-Health, vol. 19, no. 4, pp. 305-311, 2013.

[22] C. S. Gadd and L. E. Penrod, "Assessing physician attitudes regarding use of an outpatient EMR: a longitudinal, multipractice study," in Proceedings of the AMIA Annual Symposium, p. 194, Washington, DC, USA, November 2001.

[23] K. D. Kleiner, R. Akers, B. L. Burke, and E. J. Werner, "Parent and physician attitudes regarding electronic communication in pediatric practices," Pediatrics, vol. 109, no. 5, pp. 740-744, 2002.

[24] N. Almotiri, Teleconsultation perspective for cardiovascular patients in Saudi Arabia [Ph.D. thesis], Brunel University, School of Information Systems, Computing and Mathematics, 2012.

[25] M. K. A. Ghani, M. M. Jaber, and N. Suryana, "Barriers faces telemedicine implementation in the developing countries: toward building Iraqi telemedicine framework," Journal of Engineering and Applied Sciences, vol. 10, no. 4, pp. 1562-1567, 2015.

[26] A. A. Abera, G. H. Mengesha, and P. F. Musa, "Assessment of Ethiopian health facilities readiness for implementation of telemedicine," Communications of the Association for Information Systems, vol. 34, article 67, 2014.

[27] R. M. Baroud, "How ready are the stakeholders in the Palestinian healthcare system in the Gaza strip to adopt e-health?" Dissertation Abstracts International Section B: The Sciences \& Engineering, vol. 69, no. 4, 2008.

[28] F. Jing and Z. Pengzhu, "A field study of G2G information sharing in Chinese context based on the Layered behavioral model," in Proceedings of the 42nd Annual Hawaii International Conference on System Sciences (HICSS '09), pp. 1-13, Big Island, Hawaii, USA, January 2009.

[29] T. Ariyachandra and H. Watson, "Key organizational factors in data warehouse architecture selection," Decision Support Systems, vol. 49, no. 2, pp. 200-212, 2010.

[30] X. Lu, F. Liu, and R. Pei, "E-government implementation performance and information sharing: an analysis among multi-level departments," in Proceedings of the International Conference on Network Computing and Information Security (NCIS '11), vol. 1, pp. 279-283, Guilin, China, May 2011.

[31] M. A. Mohammed, A. R. Hasson, A. R. Shawkat, and N. J. Al-Khafaji, "E-government architecture uses data warehouse techniques to increase information sharing in Iraqi universities," in Proceedings of the IEEE Symposium on E-Learning, EManagement and E-Services (IS3e '12), pp. 1-5, IEEE Explore, Kuala Lumpur, Malaysia, October 2012.

[32] R. Latifi, "Ten critical steps for a successful telemedicine program," in Establishing Telemedicine in Developing Countries: From Inception to Implementation, vol. 104 of Studies in Health Technology and Informatics, pp. 60-68, IOS Press, Amsterdam, The Netherlands, 2004.

[33] S. Khoja, H. Durrani, and A. Fahim, "Scope of policy issues for eHealth: results from a structured review," in Proceedings of the Making the eHealth Connection, Bellagio, Italy, July-August 2008.

[34] N. S. Wickramasinghe, A. M. A. Fadlalla, E. Geisler, and J. L. Schaffer, "A framework for assessing e-health preparedness," International Journal of Electronic Healthcare, vol. 1, no. 3, pp. 316-334, 2005.

[35] S. Al Hasnawi, "Physicians' shortage in Iraq: impact and proposed solutions," Iraqi Journal of Community Medicine, vol. 3, pp. 214-218, 2013.
[36] L. J. Cronbach, "Response sets and test validity," Educational and Psychological Measurement, vol. 6, pp. 475-494, 1946.

[37] D. George and M. Mallery, Using SPSS for Windows Step by Step: A Simple Guide and Reference, Allyn y Bacon, Boston, Mass, USA, 2003.

[38] J. F. Hair, A. H. Money, P. Samouel, and M. Page, "Research methods for business," Education + Training, vol. 49, pp. 336337, 2007. 

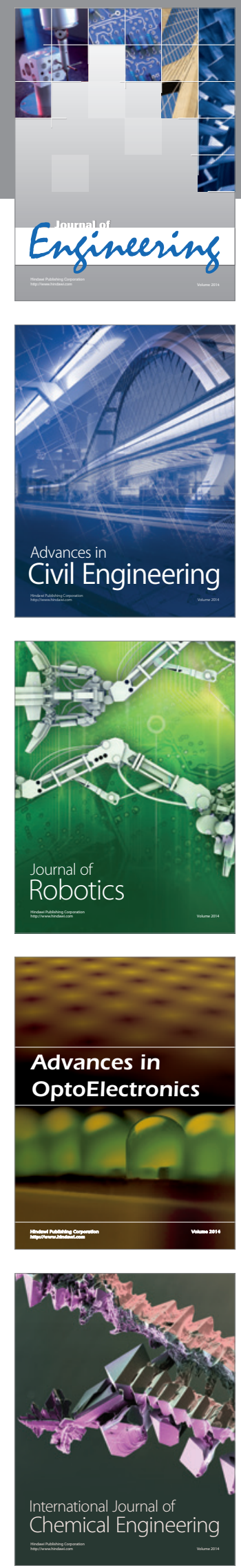

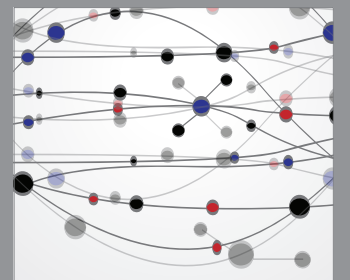

The Scientific World Journal
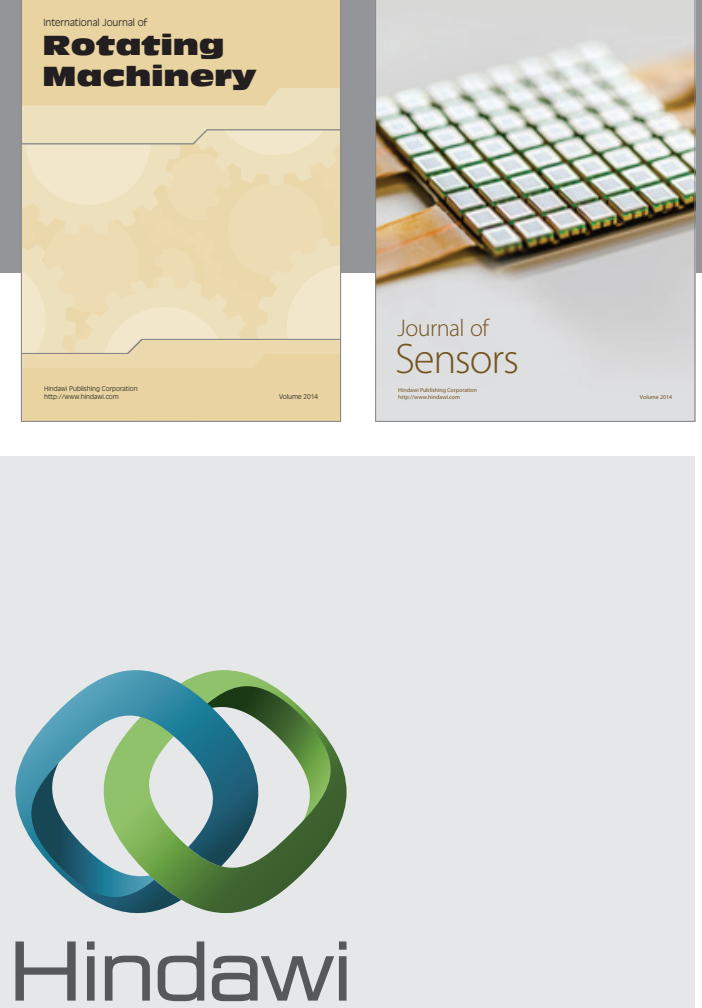

Submit your manuscripts at http://www.hindawi.com
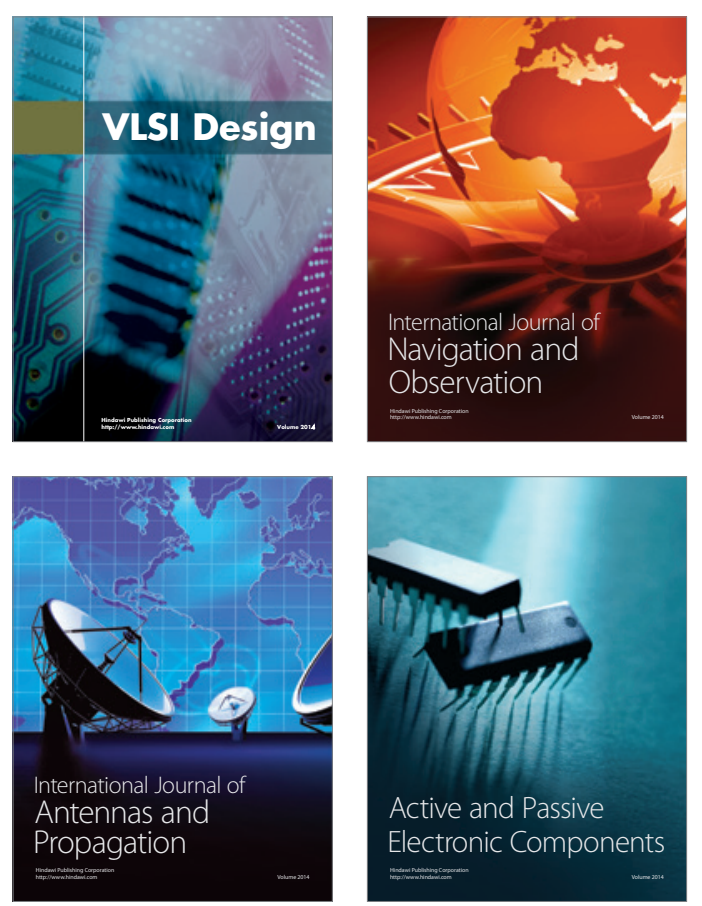
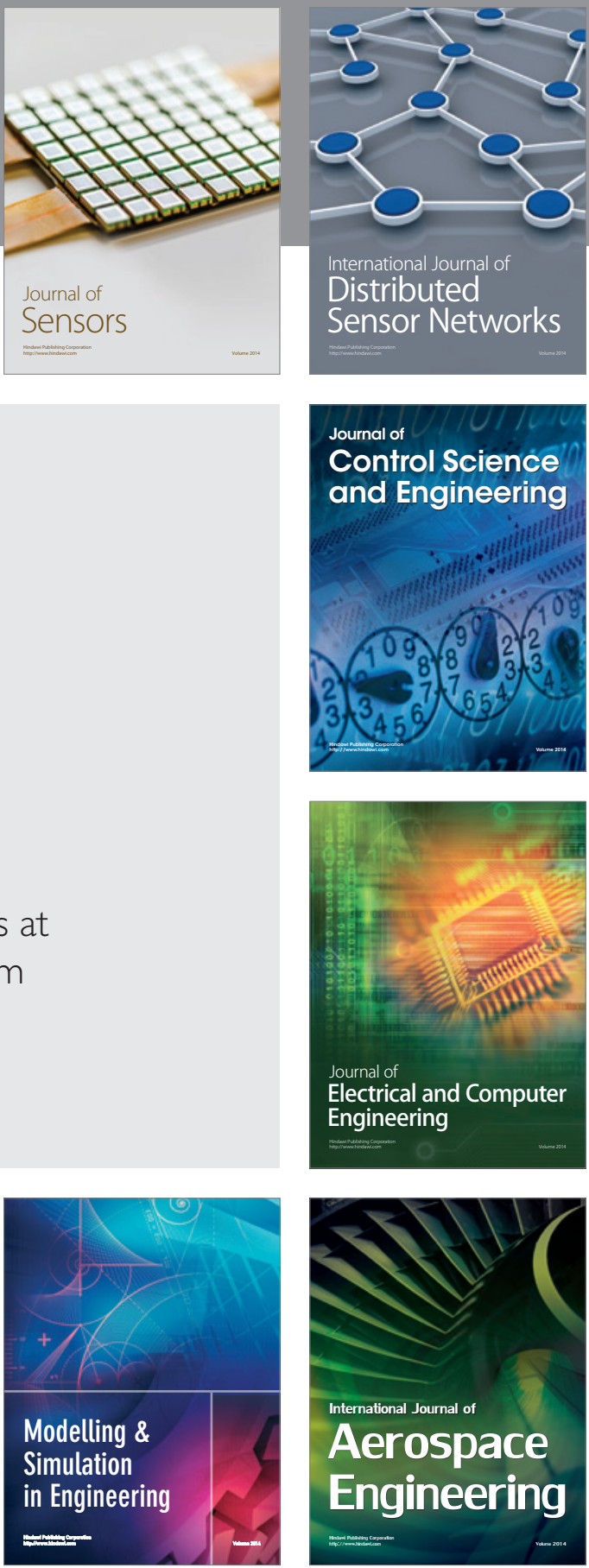

Journal of

Control Science

and Engineering
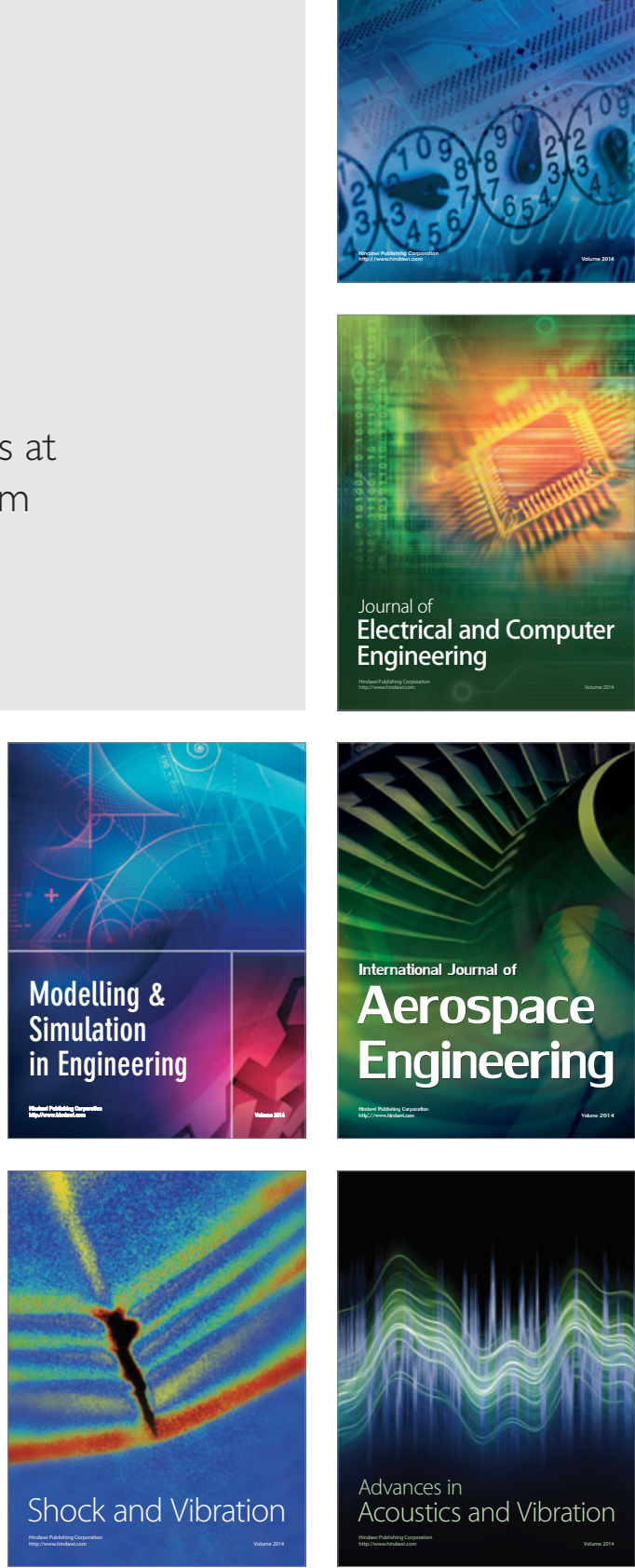